\title{
An ultrasonic humidification fluorescent tracing method for detecting unsaturated atmospheric water absorption by the aerial parts of desert plants
}

\author{
WANG Xiaohua ${ }^{*}$, XIAO Honglang, REN Juan, CHENG Yiben, YANG Qiu \\ Key Laboratory of Eco-hydrology in Inland River Basin, Cold and Arid Regions Environmental and Engineering Research \\ Institute, Chinese Academy of Sciences, Lanzhou 730000, China
}

\begin{abstract}
Atmospheric water absorption by plants has been explored for more than two centuries, and the aerial parts of plants, particularly the leaves of certain species, have been demonstrated to have an ability to absorb and utilize saturated atmospheric water such as fog, dew and condensed water. So far, however, there have been few studies on the aerial parts of desert plants in their absorption of unsaturated water from the atmosphere. This study presents an ultrasonic humidification fluorescent tracing method of detecting unsaturated atmospheric water absorption by the aerial parts of desert plants. We constructed an organic glass room based on the sizes of field plants. Then, the aboveground parts of the plants were humidified in the sealed glasshouse using an ultrasonic humidifier containing fluorescent reagents. The humidity and wetting time were controlled by turning on or off the humidifier according to the reading of a thermo-hygrometer suspended in the glasshouse. Fluorescence microscopy was employed to observe these plant samples. This method can generate unsaturated atmospheric water vapor and incorporate other fluorescent reagents or water-soluble chemical reagents for gasified humidification. In addition, it can identify plant parts that absorb unsaturated atmospheric water from the air, detect water absorption sites on the surface of leaves or tender stems, and determine the ability of tissues or microstructure of aerial parts to absorb water. This method provides a direct visual evidence for the inspection of leaf or tender stem microstructure in response to unsaturated atmospheric water absorption. Moreover, this method shows that aqueous pores in the cuticles of leaves or tender stems of desert plants are large enough to allow the passage of ionic fluorescent brightener with a molecular weight of up to $917 \mathrm{~g} / \mathrm{mol}$. Thus, this paper provides an important approach that explores the mechanism by which desert plants utilize unsaturated atmospheric water.
\end{abstract}

Keywords: ultrasonic humidification fluorescent tracer; desert plants; unsaturated atmospheric water; sites of water absorption; aqueous pores; drought environments

Citation: WANG Xiaohua, XIAO Honglang, REN Juan, CHENG Yiben, YANG Qiu. 2016. An ultrasonic humidification fluorescent tracing method for detecting unsaturated atmospheric water absorption by the aerial parts of desert plants. Journal of Arid Land, 8(2): 272-283. doi: 10.1007/s40333-015-0018-z

Atmospheric water absorption by plants has been explored for more than two centuries, and the aerial parts of plants, particularly the leaves of certain species, have been demonstrated to obtain water from the atmosphere. Many plants species have the ability to absorb and utilize fog, dew and atmospheric water via their leaves, including tomato (Brezeale et al., 1950), Pinus coulteri (Stone et al., 1950), Nolana mollis (Mooney et al., 1980), Lavandula stoechas (Munné-Bosch et al., 1999), Crassula species (Martin and Willert, 2000), Juniperus monosperma (Breshears et al., 2008), redwoods (Limm and Dawson, 2010), Bassia dasyphylla (Zhuang and Ratchiffe, 2012) and tropical

\footnotetext{
*Corresponding author: WANG Xiaohua (E-mail: wangxiaohua950@126.com) Received 2015-07-15; revised 2015-10-08; accepted 2015-10-12

(C) Xinjiang Institute of Ecology and Geography, Chinese Academy of Sciences, Science Press and Springer-Verlag Berlin Heidelberg 2016
} 
montane cloud forest plants (Eller et al., 2013; Gotsch et al., 2014).

Water absorption by the aerial parts of plants has been reported in many studies, while there has been little investigation regarding the processing of water absorption by the aerial parts of desert plants. Desert plants are widely distributed in the arid region of Northwest China. These desert plants grow under drought conditions with an average annual precipitation of less than $200 \mathrm{~mm}$, which can be less than $10 \mathrm{~mm}$ in an extremely drought year, with a typical evaporation up to more than 3,000 $\mathrm{mm}$ and with dry soils and deep underground water level that the roots of most of the shrubs and herbs cannot reach. It is impossible for desert plants to perform their life cycle, from spring-sprouting leaves to blossoms and fruit in autumn, under such conditions because of the limited annual precipitation. Is there another way for desert plants to absorb atmospheric water through their leaves to supplement their water use requirements? In general, when plants grow in desert regions, there is a strong temperature difference between day and night, and the relative humidity can be as much as $60 \%-70 \%$ during the night and the morning on a clear summer day. Do the aerial parts of desert plants absorb water from these sources?

In addition, desert plant living in arid or saline habitats has unique morphological or anatomical characteristics of their leaves or stems, such as leaves are generally degraded; some leaves have trichomes or verruca or papillae; some have salt glands and some have specialized stomata on the leaf surface. Do these specialized features on leaf surface of desert plants facilitate the absorption of water from the unsaturated atmosphere? Several methods including the isotope tracer method (Dawson and Ehleringer, 1991; Phillips and Koch, 2002; Phillips et al., 2005; Asbjornsen et al., 2007), heat ratio method (Burgess et al., 1998; Burgess and Dawson, 2004; Oliveira et al., 2005) and fluorescent dye tracer method (Strugger, 1939; Fahn, 1986; Eichert and Burkhardt, 2001; Liu Gaskin, 2004; Schreiber, 2005; Eichert et al., 2008; Eller et al., 2013) are used to identify the aerial parts of plants that absorb fog, dew and atmospheric water. The isotope tracer method overcomes the difficulty of quantifying absorption, and the heat ratio method can be used to rapidly obtain the sap flow in the field. However, these methods do not allow for visual detection and do not detect water absorption sites on the leaf surface. The fluorescent dye tracer method overcomes these limitations and provides a simple and direct visual detection; therefore, it is frequently used. In traditional saturated fluorescent humidifying method, plant leaves are immersed in an aqueous solution containing a fluorescent reagent (Strugger, 1939; Mastroberti and de Araujo Mariath, 2008; Eller et al., 2013) or are floated in the solution (Fahn, 1986; Schönherr, 2006). An alternative method is to directly spray (Grammatikopoulos and Manetas, 1994; Munné-Bosch and Alegre, 1999; Munné-Bosch et al., 1999; Gouvra and Grammatikopoulos, 2003) or smear (Eichert and Burkhardt, 2001; Liu and Gaskin, 2004; Schlegel et al., 2005; Schreiber, 2005; Eichert et al., 2008) the aqueous solution containing the fluorescent reagents on the surface of the isolated plant leaves.

However, these techniques have several limitations: (1) dye is applied to isolated plant leaves, which is suitable for indoor experiments but not applicable in the field; (2) dye can only be used for saturated wetting experiments of plant leaves and cannot verify whether the aerial parts of the plants can absorb unsaturated atmospheric water; (3) direct spraying and smearing techniques cause excessive amounts of deposition of the fluorescent reagent on the leaf surface, which increases the difficulty of identifying locations where plant leaves absorb water; and (4) the fluorescent reagents are not applied in a gasifying process. Thus, the aqueous fluorescent reagent solution is not fully utilized when directly spray or smear to the leaves or young stems, and spraying and smearing techniques often result in the waste of expensive fluorescent reagent, with a significant proportion lost during the process. In addition, covering the surface area of a large leaf is time and energy intensive and cannot always be completed.

The aim of this study was to address the above problems and to overcome the defects of the existing technologies by using ultrasonic humidification fluorescent tracer method in field. We constructed an organic glass room, then directly wetted the aerial plant parts using an ultrasonic humidifier before finally using fluorescence microscopy on plant samples. This wetting fluorescent tracer method can be used directly to identify whether atmospheric water absorption occurs, observe the microstructure on the leaf surface that is used to absorb atmospheric water and compare the ability of different structures to absorb water. 


\section{Materials and methods}

\subsection{Study area and sample selection}

The study area was located in the desert steppes of Gaolan county of Gansu province, Alxa Left Banner and Ejin Banner of Inner Mongolia, Northwest China. Gaolan county is a semi-arid area with an average precipitation of less than $400 \mathrm{~mm}$; Alxa Left Banner has an average annual precipitation of less than $200 \mathrm{~mm}$; and Ejin Banner is part of an extremely arid area with an average annual precipitation of less than $50 \mathrm{~mm}$. The dominant plant species in these regions are shrubs, half-shrubs and forbs, such as Reaumuria soongorica, Zygophyllum xanthoxylum, Haloxylon ammodendron, Nitraria tangutorum, Ammopiptanthus mongolicus and Hedysarum scoparium. In each of the three sites, plots $(1,000 \mathrm{~m} \times 1,000 \mathrm{~m})$ of desert grassland were selected and roots were sampled at depths where the plants do not receive groundwater. From June to October in 2013 and 2014, field experiments of fluorescent wetting were conducted on desert plants within the study plots.

\subsection{Saturated spraying humidification method (SHM)}

For evaluating the anatomical pathways involved in foliar water uptake, we performed saturated humidification by directly spraying plant leaves in the field plots as a preliminary experiment. Based on the results of this preliminary experiment, we subsequently conducted unsaturated atmospheric experiments by wetting the aboveground parts of the plants in a sealed glasshouse using an ultrasonic humidifier.

For the spraying experiment, two $H$. ammodendron branches with similar growth and lush leaves were sprayed with an aqueous solution of a $0.1 \%$ fluorescent brightener (FB), which is an apoplastic tracer (Fahn, 1986) that binds to the polysaccharides of cell walls and emits a strong pure blue fluorescence under an excitation wavelength of $350 \mathrm{~nm}$ (O'Brien and McCully, 1981; Munné-Bosch et al., 1999). Spraying was initiated at 18:00 and repeated once per hour to keep the leaves wet. The sprayed branches were covered with a plastic bag and sealed with parafilm at the contact between the branch and bag immediately after spraying, thus forming a moist chamber and preventing water loss. We collected samples of mature leaves at 0, 2, 4 and $6 \mathrm{~h}$ after spraying.

\subsection{Unsaturated ultrasonic humidification method (UHM)}

\subsubsection{Preparing organic glass plates}

Prior to the field wetting experiment, we customized 50 organic glass plates $(0.5 \mathrm{~m} \times 0.5 \mathrm{~m}$ or 0.25 $\mathrm{m} \times 0.5 \mathrm{~m}$, length $\times$ width). Four edges of the plates were wrapped up with a 2-cm stainless steel laminate, and a 3-mm hole was punched at $0.1 \mathrm{~m}$ from the vertex on the edge of each glass plate. Adjacent glass plates were affixed with screw caps, screw rods and screw pads. The corner of the glasshouse was affixed to two glass plates $(0.25 \mathrm{~m} \times 0.5 \mathrm{~m}$, length $\times$ width $)$ connected by hinge screws. When not in use, the two corner glass plates were folded; when in use, they were opened to form a $90^{\circ}$ angle.

\subsubsection{Building the control room}

Customized glass plates $(0.5 \mathrm{~m}$ (or $0.25 \mathrm{~m}) \times 0.5 \mathrm{~m}$ ) with connected corner glass plates were used to build glass rooms that corresponded to the plant size. For example, if a plant crown was $1.2 \mathrm{~m} \times 0.8$ $\mathrm{m}$ (length $\times$ width) in the field, we built an organic glass room that was $1.5 \mathrm{~m} \times 1.5 \mathrm{~m} \times 1.0 \mathrm{~m}$ (length $\times$ width $\times$ height) to control the wetting process. We covered the plant in the organic glass plate control room and sealed all of the gaps between the plates with transparent adhesive tape. A glass door $(0.5 \mathrm{~m} \times 0.5 \mathrm{~m})$ was installed that could be freely opened and closed for easy sampling. For this experiment, we used $Z$. xanthoxylum and $H$. ammodendron, which are species that grow well under the experimental conditions, and a glass room was built for both species for the fluorescent wetting experiment.

\subsubsection{Thermo-hygrometer placement}

A portable thermo-hygrometer MicroLog PRO-EC750 (Fourier Systems Ltd., Israel) was placed in the middle of the plant canopy within a control room to monitor the temperature and humidity of 
the glasshouse. Another portable thermo-hygrometer was simultaneously hung on a branch at the same height to monitor changes in temperature and humidity in natural environment. The thermo-hygrometers recorded temperature and humidity data once per minute, and these data were corrected according to a small artificial weather station.

\subsubsection{Ultrasonic humidifier placement}

An ultrasonic humidifier was placed on the glass plate at the top of the glass room or on a horizontal object at the side of the glass room. A small hole was made in the glass plate near the humidifier, and the humidifying tube was placed deep into the glass room. The junction between the humidifier caliber and the humidifying pipeline upper port and the joints between the humidifying pipelines below the port were also sealed with transparent adhesive tape. The thermo-hygrometer was placed far from the humidifier outlet position.

\subsubsection{Wetting control and sampling}

For creating a $0.1 \%$ fluorescent tracer solution, we weighted and dissolved $1 \mathrm{~g}$ of FB dye powder into $1 \mathrm{~L}$ of deionized water. The solution was placed in the ultrasonic humidifier, which was turned on to begin humidifying the plant samples. The changes in humidity were recorded by the thermo-hydrometer in the glass room. When the humidity reached a certain level, the humidifier was turned off. Once the humidity dropped, the humidifier was once again turned on. By carefully observing the changes in humidity with the thermo-hydrometer in the glass room, the humidity was easily maintained between the environments and the saturated atmosphere.

Several ultrasonic unsaturated wetting experiments were conducted using plants in the field. In September 2013, Experiment 1 was conducted in Luanjintan town of the Alxa Left Banner region to test field fluorescent wetting on the plant species of $Z$. xanthoxylum and $H$. ammodendron. The weather was cloudy and the environmental humidity was high on the wetting day. Although the humidity in the wetting room was high, a large difference was not observed in the humidity inside and outside of the wetting room. In fact, the concentration of fluorescent reagent in the wetting room was not high; therefore, wetting was sustained for $18 \mathrm{~h}$, continuing the second day. Subsequently, the humidifier was turned off and the glass door was opened to allow plants to exchange gas with the environment outside of the glasshouse. At 16:30 in afternoon, we continued fluorescent wetting for another $18 \mathrm{~h}$. Samples were collected at the beginning $(0 \mathrm{~h})$ and end of wetting (18 and $36 \mathrm{~h}$ ). The indoor and outdoor temperature and humidity changes were shown in Fig. 1.

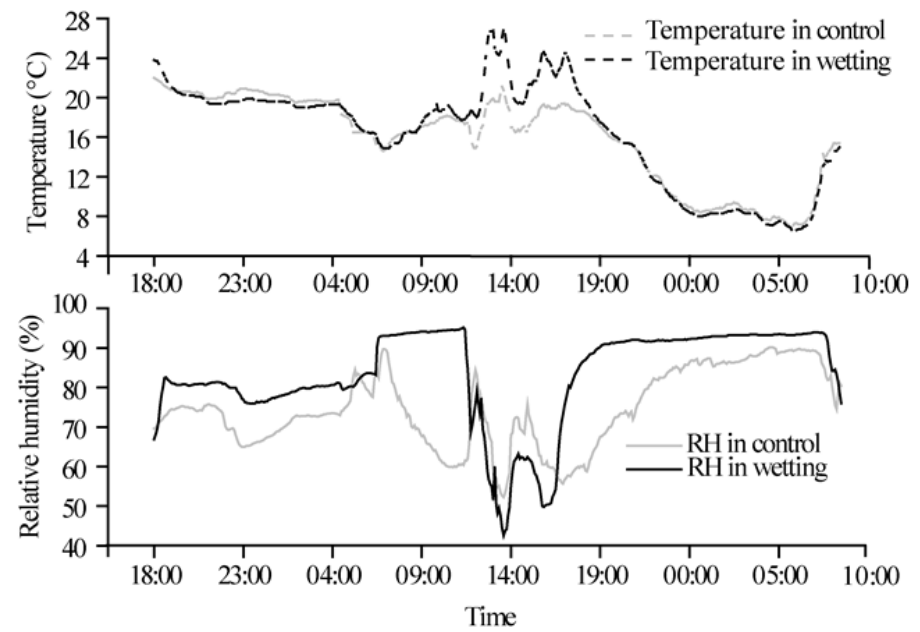

Fig. 1 The changes in temperature and relative humidity $(\mathrm{RH})$ of the exterior and interior of the glasshouse in Experiment 1 using the ultrasonic humidification method (UHM)

Experiment 2 was conducted in Lanzhou in October 2014 to test field fluorescent wetting on $H$. ammodendron. Wetting occurred from 16:30 to 21:15 and samples were collected at the beginning and end of wetting. Indoor and outdoor temperature and humidity changes were shown in Fig. 2. 

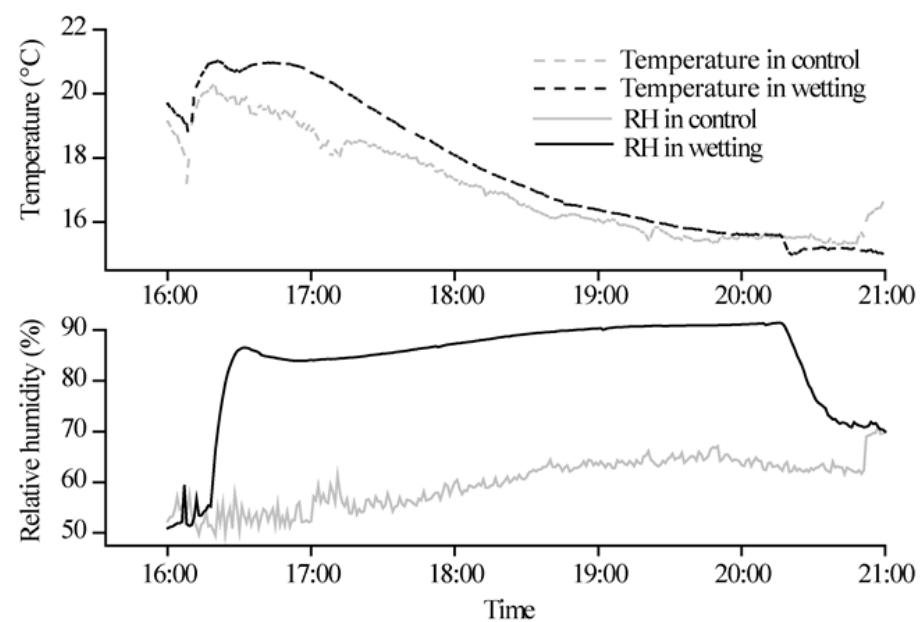

Fig. 2 The changes in temperature and relative humidity $(\mathrm{RH})$ in glasshouse and natural environment of Experiment 2 using the ultrasonic humidification method (UHM)

Experiment 3 was conducted at the end of July 2014 to test field fluorescent wetting on $H$. ammodendron. In this experiment, the wetting humidity was reduced, time interval of each sampling period was shortened, and multiple wetting and sampling events were performed. The temperature and humidity changes of Experiment 3 were shown in Fig. 3.

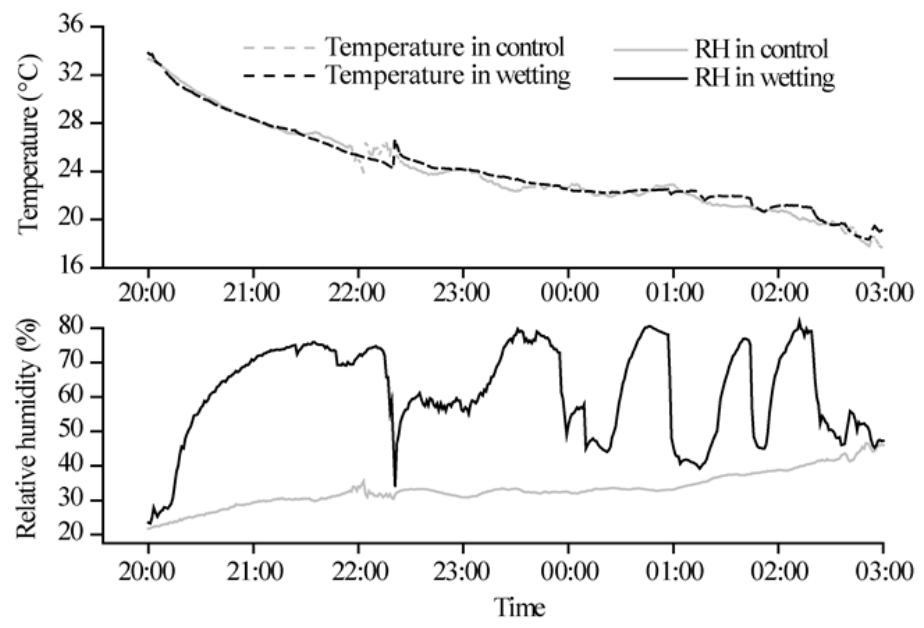

Fig. 3 The changes in temperature and relative humidity $(\mathrm{RH})$ in glasshouse and natural environment in Experiment 3 using the ultrasonic humidification method (UHM)

Mature leaf samples of Z. xanthoxylum and assimilating branch samples of $H$. ammodendron were collected. After each collection, the samples were carefully dried with filter paper, placed in a car refrigerator at approximately $4^{\circ} \mathrm{C}$, and transported to the laboratory for fluorescence detection.

\subsection{Detection of fluorescence}

Three to five mature leaves in the middle of branches were picked and washed three times with distilled water, and leaves were sectioned from the middle using a small plant tissue special slicer MTH-1 (DL Nature gene Life Sciences, Inc., Japan), whereas the epidermis was removed. All of the leaves were then mounted in glycerol and observed under an Olympus BX53 fluorescence microscope (Olympus Corporation, Tokyo, Japan) under fluorescent lighting with an excitation wavelength of $350 \mathrm{~nm}$ and occasionally under a bright field microscope. The experiment was 
repeated five to ten times, and an average of 500 randomly selected mesophyll cells were examined for each species.

\section{Results and discussion}

\subsection{Identifying atmospheric water absorption by leaves or assimilating branches}

For illustrating the beneficial effects of the gasified humidification fluorescent tracer method, we compared cross sections of the treatments and control groups of both $Z$. xanthoxylum leaves and $H$. ammodendron assimilation branches. For Experiment 1, the relative humidity and temperature inside and outside of the organic glass room were shown in Fig. 1, and the results of the comparison were shown in Figs. 4 and 5. Figures 4a and d and Figs. 5a and d represent the control groups, and Figs. 4b, c, e and f and Figs. 5b, c, e and f represent the treatments. The chloroplasts of mesophyll cells in the control groups contained a large amount of chlorophyll, which emits red light (Figs. 4a and d; Figs. 5a and d) at $350 \mathrm{~nm}$ wavelength, whereas the chloroplasts of mesophyll cells in treatments all emitted blue fluorescence to varying degrees (Figs. 4b, c, e and f; Figs. 5e and f) because FB is an apoplastic tracer (Fahn, 1986) that binds to the polysaccharides of the cell wall and emits a strong pure blue fluorescence under $350 \mathrm{~nm}$ wavelength (O’Brien and McCully, 1981; Munné-Bosch et al., 1999). Our results indicated that $Z$. xanthoxylum leaves and $H$. ammodendron assimilation branches absorbed atmospheric water to different degrees during atmospheric wetting. At $18 \mathrm{~h}$ of wetting, the mesophyll cells of the upper epidermis and the lower epidermis of $Z$. xanthoxylum leaves emitted the same intensity of pure blue fluorescence (Figs. 4e and $\mathrm{f}$ ), which indicates that the upper epidermis and the lower epidermis $Z$. xanthoxylum leaves had the same ability to absorb water. After $36 \mathrm{~h}$ of wetting, Z. xanthoxylum leaves and H. ammodendron assimilation branches reached higher absorption values and showed strong pure blue fluorescence (Fig. 4c; Figs. 5c and f). All of the mesophyll cells of Z. xanthoxylum leaves changed from red (Figs. 4a and d) to blue (Fig. 4c), while only a part of mesophyll cells of the assimilating branches of $H$. ammodendron changed from red to blue (Fig. 5f). At the same time, most of water storage tissues (WST) of the assimilating branches of $\mathrm{H}$. ammodendron changed from light blue (Fig. 5a) or grey white (Fig. 5d) to pure blue (Figs. 5c and f). These findings suggest that the water-absorbing ability of $Z$. xanthoxylum leaves is stronger than those of $H$. ammodendron assimilating branches, and they also suggest that atmospheric water absorbed by the leaves of $Z$. xanthoxylum is mainly stored in mesophyll cells and that water uptake by young branches of $H$. ammodendron is mainly stored in water storage tissues. Our results indicated that this fluorescent wetting method is capable of identifying leaves and young branches that can absorb atmospheric water from unsaturated atmospheric air and of evaluating the ability of tissues or structures to absorb water by comparing the intensity of pure blue fluorescence.

The ability of $Z$. xanthoxylum leaves and $H$. ammodendron assimilating branches to absorb water from unsaturated atmospheric air may be related to their micro-morphologic structures and surface chemistry. Secreted hydrophilic compounds (Koch et al., 2009) or deposited pollutants and aerosols (Burkhardt et al., 2012) can effectively adsorb water to the leaf surface or condense water molecules on leaves even in unsaturated atmospheres (Mooney et al., 1980). Neinhuis and Barthlott (1997) found that plant hydrophilic surfaces are relatively smooth cell surfaces (tabular and slightly convex cells) without three-dimensional waxes. However, super hydrophobic plant surfaces are water repellent and formed by micro-structured cells superimposed with three-dimensional waxes. Plants with these leaf surfaces include lotus (Nelumbo nucifera) (Barthlott and Neinhuis, 1997; Neinhuis and Barthlott, 1997; Bhushan and Jung, 2006; Burton and Bhushan, 2006), rice (Oryza sativa) and parrot feather (Myriophyllum aquaticum). Certain plants alternatively possess super hydrophobic multi-cellular hairs (Otten and Herminghaus, 2004). 


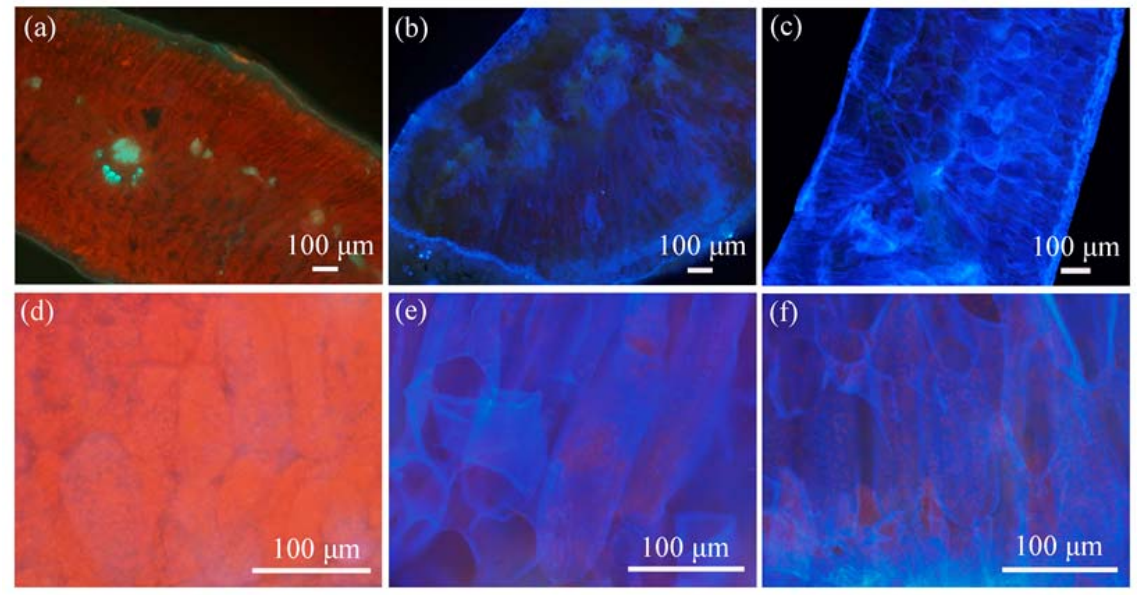

Fig. 4 Cross-sections and mesophyll cells of the leaves of Z. xanthoxylum at different wetting times in Experiment 1. Cross section of wetting at 0 (a), 18 (b) and 36 h (c) $(\times 100)$; mesophyll cells at 0 h of wetting (d) $(\times 400)$; mesophyll cells of the upper epidermis (e) and lower epidermis (f) at $18 \mathrm{~h}$ of wetting $(\times 400)$.

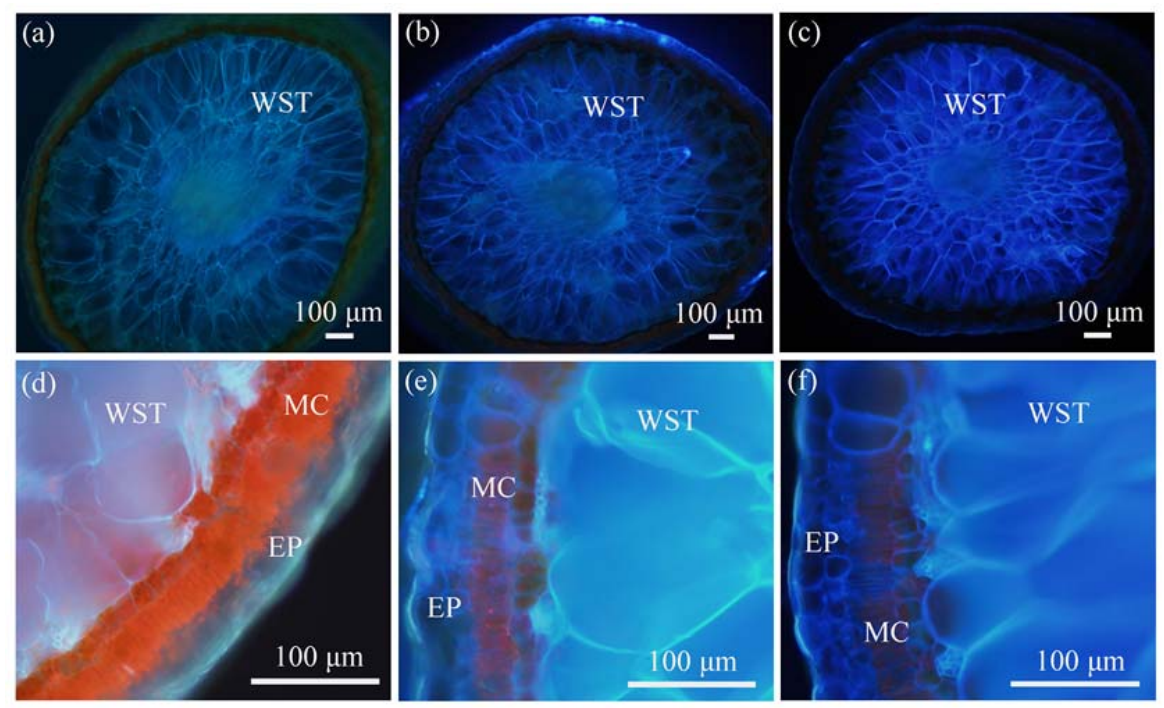

Fig. 5 Cross-sections of the assimilating branches of $H$. ammodendron at different wetting times in Experiment 1. Cross-sections of wetting at 0 (a), 18 (b) and 36 h (c) (×100); cross-sections of wetting at 0 (d), 18 (e) and 36 h (f) $(\times 400)$. WST means water storage in tissue; MC, mesophyll cells; EP, epidermis.

\subsection{Identification of absorption sites}

The ability of the aerial parts of desert plants to absorb water from unsaturated atmospheric air may be related to their micro-morphologic structures. For further identifying the absorption sites or micro-morphologic structures for adsorbing atmospheric water on the leaf surface, we conducted Experiments 2 and 3, in which the sampling time intervals were shortened and the humidity of the fluorescent wetting was reduced. Figures $6 a$ and $b$ showed the fluorescent surface of a sample of $H$. ammodendron assimilating branches collected during Experiment 2. Figures 6c and d and Figs. 7a and $b$ showed the fluorescent surfaces and cross sections, respectively, from Experiment 3 samples collected at 00:55. As seen in Figs. 6a and c, certain patches emitted strong blue fluorescent light, and they represented the stomata observed in the corresponding light photographs in Figs. 6b and d. Similarly, the fluorescent cross-sections of an assimilating branch of $\mathrm{H}$. ammodendron showed a similar phenomenon (Fig. 7), with only certain stomata producing strong blue fluorescent light. In Figs. 6a and c and Fig. 7a, the production of strong blue fluorescent light by the stomata indicates 
these stoma are activated and can absorb unsaturated atmospheric water; conversely, the lack of blue fluorescent light indicates that these stomata aren't activated and they cannot absorb water, i.e. $H$. ammodendron assimilating branches first capture atmospheric water by partially activated stomata (Figs. 6a and c) and atmospheric water enters mesophyll cells via these activated stomata (Fig. 7a). Burkhardt (2010) referred to this phenomenon as the 'hydraulic activation of stomata' (HAS).

Activated stomata may result from stomata mucilage (Zimmermann et al., 2007; Westhoff et al., 2009) or hygroscopic atmospheric depositions (Burkhardt, 2010) on or around the stomata, which changes the leaf surface around the stomata from hydrophobic to hydrophilic. Therefore, these activated stomata have the ability to first adsorb atmospheric water and change it into mesophyll cells. Our results are consistent with those of Eichert et al. (2008) and Burkhardt et al. (2012), who used more advanced and complex operation methods. Eichert et al. (2008) visualized the stomatal uptake of fluorescent nanoparticles (43 nm in diameter) via confocal laser scanning microscopy, and Burkhardt et al. (2012) confirmed the stomatal uptake of aqueous solutions and noted that humidity fluctuations promoted the spatial expansion of salts into the stomata, which was observed using an environmental scanning electron microscope.
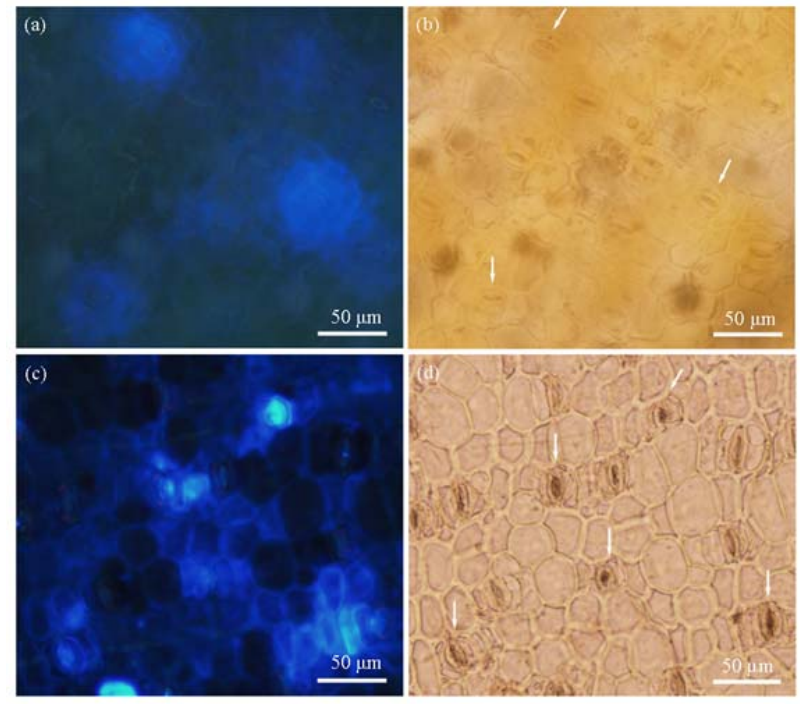

Fig. 6 Epidermis structure (ES) of assimilating branches of $H$. ammodendron at different fluorescent wetting times in Experiments 2 and 3. (a) ES of Experiment 2 under fluorescence at 4 h of wetting; (b) corresponding to ES under bright light; (c) ES of Experiment 3 under fluorescence at sampling 00:55 (i.e. after the third wetting and the total wetting time is approximately $3 \mathrm{~h}$ ); (d) corresponding to ES under bright light. Arrows point to the stomata.
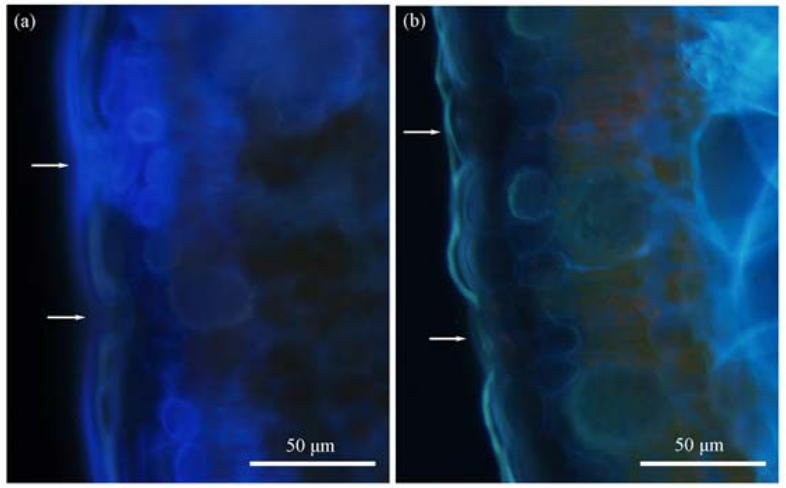

Fig. 7 Cross-section of an assimilating branch of $H$. ammodendron in Experiment 3 using the UHM. (a) sampling at 00:55; (b) sampling at 20:00 (i.e. at the beginning of wetting $0 \mathrm{~h}$ ). Arrows point to the stomata. 
Figure 8 showed fluorescent photographs of $H$. ammodendron assimilating branches that were subjected to the saturated spraying humidification method and collected after $2 \mathrm{~h}$ of wetting. However, because excess fluorescent reagents occurred at the surface, the photographs could not be used to pinpoint the first absorbing water sites of $H$. ammodendron assimilating branches despite the short sampling time.
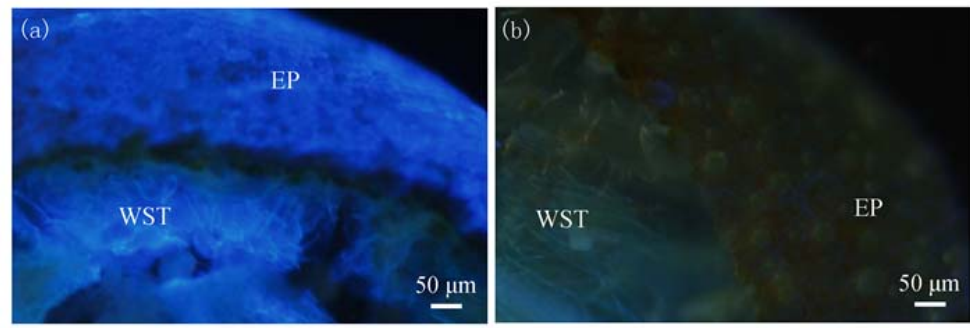

Fig. 8 Cross-section of an assimilating branch of H. ammodendron treated with the SHM. (a) spraying at 2 h; (b) spraying at $0 \mathrm{~h}$. WST means water storage in tissue; EP, epidermis.

The ultrasonic fluorescent humidification method can be used to identify surface water absorption sites of assimilating branches of $H$. ammodendron, whereas the spraying or smearing methods do not provide accurate identifications. By using fluorescent reagents in a gasifying process, we can conveniently maintain appropriate humidity levels, wetting intervals and fluorescent reagent solution concentrations to control the amount of fluorescence absorbed by the leaves. Consequently, we can easily detect small amounts of fluorescence on leaf surfaces and thus identify the absorbing water sites on plant leaves. The direct spraying or smearing method applies an excess of fluorescent reagents on the surfaces of leaves or young branches, which often results in non-water absorption sites displaying fluorescence. Therefore, these two methods are not suitable for the identification of water absorption sites.

It is easy to identify that stomata are the surface water absorption sites of assimilating branches of $H$. ammodendron using the ultrasonic fluorescent humidification method, but the stomata are not the only sites for water absorption for desert plants. In fact, using the fluorescent humidification method, we identified that the surface water absorption sites of the aerial parts of desert plants are different, of which different desert plant species are different, and sometimes even different growing seasons of the same species, of which are also different. All these results will be reported in the future articles in detail.

\subsection{Evaluation of fluorescent reagent consumption}

In the ultrasonic wetting method, the amount of applied fluorescent reagents was much lower than that used during the spraying experiment. The ultrasonic wetting method vaporized aqueous reagents into gas or fog, which allows the entire plant leaf or young branch to be fully exposed to the fluorescent reagent. This feature results in the efficient use of these expensive fluorescent reagents, thus limiting the amounts that fall to the ground and are wasted. In addition, ultrasonic wetting is simple and easily conducted compared with the smearing method, which is time and energy intensive. Finally, ultrasonic wetting may also reduce the random errors that result from spraying, which may unevenly distribute fluorescent reagents.

\subsection{Assessment of aqueous pores of the desert plant cuticle}

Based on the experimental verification of several typical desert plants, including $R$. soongarica, Tamarix ramosissima, Zygophyllum xanthoxylum, $H$. ammodendron, Nitraria tangutorum, Ammopiptanthus mongolicus, Cynanchum hancockianum and Hedysarum scoparium, we also demonstrated that the aerial parts of desert plants can absorb unsaturated atmospheric water and the aqueous solutes FB with molecular weights up to $917 \mathrm{~g} / \mathrm{mol}$ can be gasified together with water molecules. The fluorescent dye tracer FB was adsorbed by the cuticles of the aerial parts of plants and ultimately passed through aqueous pores of the plant cuticles into mesophyll cells. This is the first study to present the fluorescent dye tracer FB with a molecular weight of $917 \mathrm{~g} / \mathrm{mol}$ to trace 
the path of water from the leaf surface to mesophyll cells.

Recent studies on the ecological function of leaf surface cuticle, where aqueous pores are present, have been updated. When the air is very dry, pores shrink or disappear and water permeability decreases. This helps leaves retain water. When the air humidity is high such as in fog or rain, cuticles swell and form water-soluble or ion passages-aqueous pores that allow leaves or tender stems to take up water (Schönherr, 2006). In addition, the size of aqueous pores in surface cuticles of plant species is different and their permeability and their size selectivity for ionic particles are also different. The cuticular ledges of guard cells of Helxine (Strugger, 1939), Rhoeo discolor, Hydrocleys nymphoides (Bauer, 1953), Vicia faba and Commelina communis (Maier-Maercker, 1979) have aqueous pores large enough to allow the passage of ionic berberine sulphate (BS) with molecular weights up to $768.8 \mathrm{~g} / \mathrm{mol}$. In contrast, the astomatous Citrus spp. were practically impermeable to saccharose $(342 \mathrm{~g} / \mathrm{mol})$ and raffinose $(505 \mathrm{~g} / \mathrm{mol})$, which were excluded from the aqueous pores by virtue of their sizes (Schönherr, 1976).

The fluorescent dye tracer FB used in this study is a soluble ionic fluorescent dye with a molecular weight up to $917 \mathrm{~g} / \mathrm{mol}$. The aerial parts of desert plants allow the passage of ionic FB from the leaf surface to the mesophyll cells, which indicates that the surface cuticles of water absorption sites of desert plants which have aqueous pores that are large enough to allow the passage of ionic FB with molecular weights up to $917 \mathrm{~g} / \mathrm{mol}$. These characteristics of desert plants with large aqueous pores in the cuticle of water absorption sites of leaves or tender stems may be more favorable for the absorption and utilization of atmospheric water, enabling desert plants with strong drought resistance to survive in extremely arid environments.

\section{Conclusions}

The ultrasonic humidification fluorescent tracer method presented here provides the following benefits over currently applied techniques. (1) The leaves of plants can be humidified in the field. (2) The method can identify the plant parts that absorb water from an unsaturated atmosphere, detect water absorption sites at the leaf surface and compare the ability of tissues or microstructures to absorb water. (3) The method can gasify hydrophilic reagents with larger molecular weights (917 $\mathrm{g} / \mathrm{mol}$ ) can penetrate the leaf or tender stem surfaces into mesophyll cells. This indicates that there are large aqueous pores in the surface cuticles of leaves or tender stems of desert plants. This method is preferable to explore the mechanism desert plants utilizing unsaturated atmospheric water.

\section{Acknowledgments}

This study was supported by the National Natural Science Foundation of China (91125025, 31400319).

\section{References}

Asbjornsen H, Mora G, Helmers M J. 2007. Variation in water uptake dynamics among contrasting agricultural and native plant communities in the Midwestern U.S. Agriculture, Ecosystem \& Environment, 121(4): 343-356.

Barthlott W, Neinhuis C. 1997. Purity of the sacred lotus, or escape from contamination in biological surfaces. Planta, 202(1): 1-8.

Bauer L. 1953. Addressing the question of mass transport within the plant with specific emphasis on the migration of fluorochromes. Planta, 42(5): 367-451.

Bhushan B, Jung Y C. 2006. Micro- and nanoscale characterization of hydrophobic and hydrophilic leaf surfaces. Nanotechnology, 17(11): 2758-2772.

Breshears D D, McDowell N G, Goddard K L, et al. 2008. Foliar absorption of intercepted rainfall improves woody plant water status most during drought. Ecology, 89(1): 41-47.

Brezeale E L, McGeorge W T, Brezeale J F. 1950. Moisture absorption by plants from an atmosphere of high humidity. Plant Physiology, 25(3): 413-419.

Burgess S S O, Adams M A, Turner N C, et al. 1998. The redistribution of soil water by tree root systems. Oecologia, 115(3): 
306-311.

Burgess S S O, Dawson T E. 2004. The contribution of fog to the water relations of Sequoia sempervirens (D. Don): foliar uptake and prevention of dehydration. Plant, Cell \& Environment, 27(8): 1023-1034.

Burkhardt J. 2010. Hygroscopic particles on leaves: nutrients or desiccants? Ecological Monographs, 80(3): 369-399.

Burkhardt J, Basi S, Pariyar S, et al. 2012. Stomatal penetration by aqueous solutions—an update involving leaf surface particles. New Phytologist, 196(3): 774-787.

Burton Z, Bhushan B. 2006. Surface characterization and adhesion and friction properties of hydrophobic leaf surfaces. Ultramicroscopy, 106(8-9): 709-719.

Dawson T E, Ehleringer J R. 1991. Streamside trees that do not use stream water. Nature, 350(6316): 335-337.

Eichert T, Burkhardt J. 2001. Quantification of stomatal uptake of ionic solutes using a new model system. Journal of Experimental Botany, 52(357): 771-781.

Eichert T, Kurtz A, Steiner U, et al. 2008. Size exclusion limits and lateral heterogeneity of the stomatal foliar uptake pathway for aqueous solutes and water-suspended nanoparticles. Physiologia Plantarum, 134(1): 151-160.

Eller C B, Lima A L, Oliveira R S. 2013. Foliar uptake of fog water and transport belowground alleviates drought effects in the cloud forest tree species, Drimys brasiliensis (Winteraceae). New Phytologist, 199(1): 151-162.

Fahn A. 1986. Structural and functional properties of trichomes of xeromorphic leaves. Annals of Botany, 57(5): 631-637.

Gotsch S G, Asbjornsen H, Holwerda F, et al. 2014. Foggy days and dry nights determine crown-level water balance in a seasonal tropical montane cloud forest. Plant, Cell \& Environment, 37(1): 261-272.

Gouvra E, Grammatikopoulos G. 2003. Beneficial effects of direct foliar water uptake on shoot water potential of five chasmophytes. Canadian Journal of Botany, 81(12): 1278-1284.

Grammatikopoulos G, Manetas Y. 1994. Direct absorption of water by hairy leaves of Phlomis fruticosa and its contribution to drought avoidance. Canadian Journal of Botany, 72(12): 1805-1811.

Koch K, Bhushan B, Barthlott W. 2009. Multifunctional surface structures of plants: An inspiration for biomimetics. Progress in Materials Science, 54(2): 137-178.

Limm E B, Dawson T E. 2010. Polystichum munitum (Dryopteridaceae) varies geographically in its capacity to absorb fog water by foliar uptake within the redwood forest ecosystem. American Journal of Botany, 97(7): 1121-1128.

Liu Z Q, Gaskin R E. 2004. Visualisation of the uptake of two model xenobiotics into bean leaves by confocal laser scanning microscopy: diffusion pathways and implication in phloem translocation. Pest Management Science, 60(5): 434-439.

Maier-Maercker U. 1979. Peristomatal transpiration and stomatal movement: a controversial view III. Visible effects of peri stomatal transpiration on the epidermis. Journal of Plant Physiology, 91(3): 225-238.

Martin C E, von Willert D J. 2000. Leaf epidermal hydathodes and the ecophysiological consequences of foliar water uptake in species of Crassula from the Namib Desert in Southern Africa. Plant Biology, 2(2): 229-242.

Mastroberti A A, de Araujo Mariath J E. 2008. Development of mucilage cells of Araucaria angustifolia (Araucariaceae). Protoplasma, 232(3-4): 233-245.

Mooney H A, Gulmon S L, Ehleringer J, et al. 1980. Atmospheric water uptake by an Atacama Desert shrub. Science, 209(4457): 693-694.

Munné-Bosch S, Alegre L. 1999. Role of dew on the recovery of water-stressed Melissa officinalis L. plants. Journal of Plant Physiology, 154(5-6): 759-766.

Munné-Bosch S, Nogués S, Alegre L. 1999. Diurnal variations of photosynthesis and dew absorption by leaves in two evergreen shrubs growing in Mediterranean field conditions. New Phytologist, 144(1): 109-119.

Neinhuis C, Barthlott W. 1997. Characterization and distribution of water-repellent, self-cleaning plant surfaces. Annals of Botany, 79(6): 667-677.

O’Brien T P, McCully M E. 1981. The Study of Plant Structure: Principles and Selected Methods. Melbourne: Termarcarphi Pty. Ltd.

Oliveira R S, Dawson T E, Burgess S S O. 2005. Evidence for direct water absorption by the shoot of the desiccation-tolerant plant Vellozia flavicans in the savannas of central Brazil. Journal of Tropical Ecology, 21(5): 585-588.

Otten A, Herminghaus S. 2004. How plants keep dry: a physicist’s point of view. Langmuir, 20(6): 2405-2408.

Phillips D L, Koch P L. 2002. Incorporating concentration dependence in stable isotope mixing models. Oecologia, 130(1): 114-125.

Phillips D L, Newsome S D, Gregg J W. 2005. Combining sources in stable isotope mixing models: alternative methods. Oecologia, 144(4): 520-527.

Schlegel T K, Schönherr J, Schreiber L. 2005. Size selectivity of aqueous pores in stomatous cuticles of Vicia faba leaves. Planta, 221(5): 648-655. 
Schönherr J. 1976. Water permeability of isolated cuticular membranes: the effect of cuticular waxes on diffusion of water. Planta, 131(2): 159-164.

Schönherr J. 2006. Characterization of aqueous pores in plant cuticles and permeation of ionic solutes. Journal of Experimental Botany, 75(11): 2471-2491.

Schreiber L. 2005. Polar paths of diffusion across plant cuticles: new evidence for an old hypothesis. Annals of Botany, 95(7): 1069-1073.

Stone E C, Went F W, Young C L. 1950. Water absorption from the atmosphere by plants growing in dry soil. Science, 111(2890): 546-548.

Strugger S. 1939. Luminescence microscopical analysis of the parenchymatic plant transpiration stream. 3. Investigations on Helxine soleirolii Req. Biological Central Journal, 59: 409-442.

Westhoff M, Zimmermann D, Zimmermann G, et al. 2009. Distribution and function of epistomatal mucilage plugs. Protoplasma, 235: 101-105.

Zhuang Y L, Ratchiffe S. 2012. Relationship between dew presence and Bassia dasyphylla plant growth. Journal of Arid Land, 4(1): 11-18.

Zimmermann D, Westhoff M, Zimmermann G, et al. 2007. Foliar water supply of tall trees: evidence for mucilage-facilitated moisture uptake from the atmosphere and the impact on pressure bomb measurements. Protoplasma, 232(1-2): 11-34. 